\title{
NEURAL OUTPUT REGULATION FOR A SOLAR POWER PLANT
}

\author{
J. Henriques, P. Gil and A. Dourado \\ CISUC - Centro de Informática e Sistemas da Universidade de Coimbra, \\ Departamento de Engenharia Informática \\ Pólo II da Universidade, Pinhal de M arrocos, 3030 Coimbra, Portugal \\ e-mail: \{jh, pgil, dourado\}@dei.uc.pt, Phone: + 351239790000 Fax:+ 351239701266
}

\begin{abstract}
A bstract: In this paper the modelling capabilities of a recurrent neural network and the effectiveness and stability of the output regulation control theory are combined. The control structure consists in a neural based indirect adaptive control scheme, being the main goal to provide a viable practical control strategy suitable for real-time implementations. This control scheme was applied to the distributed solar collector field at Plataforma Solar de Almería, Spain. Experimental results obtained at the solar power plant are presented showing the effectiveness of the proposed approach.
\end{abstract}

Keywords: Recurrent neural networks, output regulation, non-linear control, on-line learning, solar power plants.

\section{INT RODUCTION}

The main control requirement in a solar power plant is to maintain the outlet oil temperature of the collector field at a constant pre-specified value. One of the main features of the plant is that its primary energy source, the solar radiation, can not be manipulated by the control system. Moreover, since the solar radiation changes substantially during plant operation, due to the daily solar cycle, atmospheric conditions such as a the cloud cover, humidity and air transparency, this leads to significant variations in the dynamics of the field (e.g. the response rate and time delay), corresponding to different operating conditions. Therefore, it is difficult to obtain a satisfactory performance over the whole operating range with a fixed linear controller.

One possibility to overcome these difficulties is to use adaptive control schemes, on the basis of local linear models of the plant, which mimic changes during the operation and are used for self-tuning or within predictive control schemes (Camacho et al, 1992; Camacho et al, 1994; Pickhardt and Silva, 1998). The distributed solar collector field is a process where the main disturbances, the solar radiation and the inlet oil temperature, are measurable. F ollowing this idea Coito et al (1997) have presented simulation and experimental results concerning the design of a predictive controller (MUSMAR), and Cardoso et al (1999) have presented a fuzzy supervisor strategy that takes into account this measured disturbances.

Others have suggested intelligent control techniques, such as neural networks (A rahal et al, 1997) or fuzzy systems (B erenguel et al, 1997), (Rubio et al, 1995), (Oksanen and Juuso, 1999). Another possible alternative could be the commissioning of a switching controller using different models of the plant for different operating points. Henriques et al (1999) have suggested a control strategy based on a PID control design with a fuzzy logic-switching supervisor. The supervisor is built upon a Takagi-Sugeno fuzzy model to implement an on-line switching between several PID controllers according to the real time measured conditions.
Recently, neural networks ( NN) have attracted a great deal of attention owing to their ability to learn nonlinear functions from input-output data examples (Cybenko, 1989). Applied to control field, NN are essentially non-linear models that can be useful to solve non-linear control problems (Hunt and Zbikowski, 1997). Due to their intrinsic abilities to incorporate time, recurrent neural networks (RNN), introduced by Hopfield (1982) and further developed by some other authors (Rovithakis and Christodoulou, 1997), (Poznyak et al, 1999), (K ulawski and Brdys, 2000) have some advantages with respect to static $\mathrm{NN}$, mainly for modelling dynamic processes purposes.

In this paper a RNN is used to replace the unknown system, transforming the original problem into a nonlinear control problem suitable to be designed by nonlinear control techniques. In this context, the geometric approach has provided a variety of tools for the analysis and design of non-linear control systems.

A well-known theory is the output regulation (OR) that aims to derive a control law such that the closed loop system is stable and, simultaneously, the tracking output error converges to zero. This technique leads to a straightforward method for solving non-linear control problems. However, the OR theory assumes a perfect model knowledge. Given the neural model plant mismatch, an on-line adaptation of neural networks weights is considered in order to improve the discrepancies between the output of a previous off-line model and the actual output of the system. By means of a Lyapunov analysis a stability condition for the weights updating is employed.

The paper is organised as follows: section 2 gives a short description of the solar power plant. In section 3 the proposed NN architecture and the associated offline and on-line learning laws are presented. The OR theory is reviewed in section 4 and the adaptive neural-control structure is introduced. In section 5 some simulation and experimental results collected from the solar power plant are presented. Finally, section 6 concludes the paper. 


\section{THE SOLAR POWER PLANT}

The Acurex distributed solar collector field at Plataforma Solar de Almería (PSA) is quite well described in available literature (Kaltz, 1982; Camacho et al, 1992) and is located at the desert of Tabernas, in south of Spain. The field consists of 480 distributed solar collectors arranged in 20 rows, which form 10 parallel loops. Each loop is $172 \mathrm{~m}$ long and the total aperture surface is $2672 \mathrm{~m}^{2}$. The plant is able to provide $1.2 \mathrm{MW}$ peak of thermal power. A schematic diagram is shown in Fig. 1.

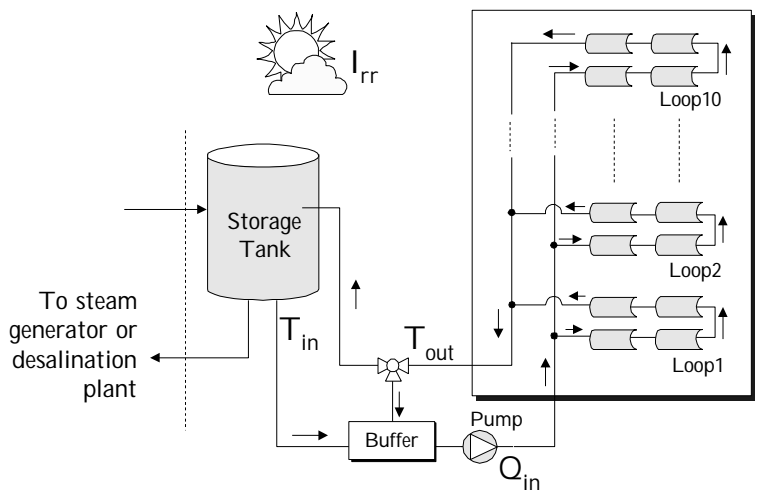

Fig. 1: Schematic diagram of the A curex field.

Each collector uses parabolic mirrors to concentrate the radiation in a receiver tube. Synthetic oil is pumped through the receiver tube and picks up the heat transferred through the tube walls. The cold inlet oil is collected from the bottom of the storage tank and is passed through the field by using a pump at the field inlet. The heated fluid is introduced into the storage tank to be used for electrical energy generation or feeding a heat exchanger of the desalination plant. The manipulated variable in the plant is the oil flow rate $Q_{i n}$ being the main goal to regulate the outlet field oil temperature $T_{\text {out }}$ at a desired value $T_{r e f}$. The main disturbances are the solar radiation $I_{r r}$ and the inlet oil temperature $T_{i n}$

\section{RECURRENT NEURAL NETWORKS}

The solar plant is assumed to be described in the form (1),

$$
\begin{gathered}
x_{p}(k+1)=f\left(x_{p}(k), u(k)\right) \\
y(k)=C x_{p}(k)
\end{gathered}
$$

where $f: \Re^{n_{p}} \mathfrak{R}^{n_{u}} \rightarrow \mathfrak{R}^{n_{p}}$ defines a non-linear function. The vector $x_{p} \in \mathfrak{R}^{n_{p}}$ is the state of the process (assumed to be unknown and inaccessible), $u \in \mathfrak{R}^{n_{u}}$ and $y \in \mathfrak{R}^{n_{y}}$ are, respectively, the process input and output.

\subsection{Proposed Recurrent Neural Architecture}

Given the approximation capabilities of RNN ( in et al, 1999) it is assumed that there exist a RNN, described by (2) and shown in Fig. 2, that is able to describe the plant's dynamics.

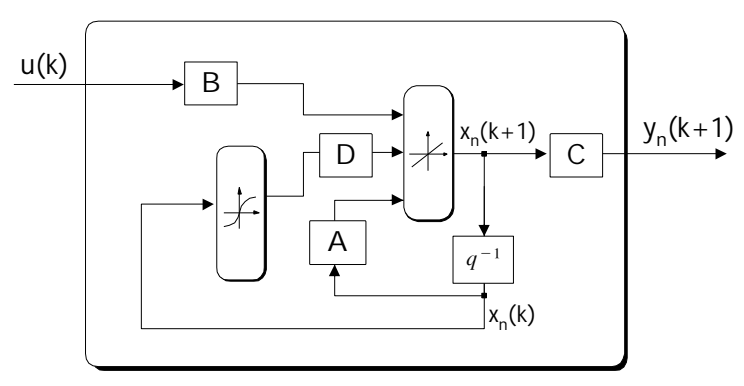

Fig. 2: Recurrent neural network structure.

$$
\begin{gathered}
x_{n}(k+1)=A x_{n}(k)+D \sigma\left(x_{n}(k)\right)+B u(k) \\
y_{n}(k)=C x_{n}(k)
\end{gathered}
$$

The vector $x_{n} \in \mathfrak{R}^{n}$ is the output of the hidden layer, known as the network hyper-state, and $y_{n} \in \mathfrak{R}^{n_{y}}$ is the network output. $A \in \mathfrak{R}^{n \times n}, B \in \mathfrak{R}^{n \times n_{u}}, C \in \mathfrak{R}^{n_{y} \times n}, D \in \mathfrak{R}^{n \times n}$ are interconnection matrices and the neural activation function $\sigma(\cdot)$ is the hyperbolic tangent function. This architecture can be seen as a modification of the original discrete time RNN proposed by Hopfield, with an additional exogenous input. On the other hand, this can be seen as a hybrid model, with a linear and a non-linear part.

\subsection{Parameter Estimation}

Off-line learning: As pointed out by Hagan and Menhaj (1994) the Levenberg-Marquardt is more efficient than other techniques when the network contains no more than a few hundred parameters. Due to its effectiveness this algorithm has been applied for the off-line training of the RNN. From this initial training phase results the network parameters, expressed in $A^{*}, B^{*}, C^{*}$ and $D^{*}$ matrices.

On-line learning: Several training algorithms have been proposed to recursively adjust the network parameters in recurrent networks. Typical examples are the real time recurrent algorithm (Williams and Zipser, 1995), the dynamic backpropagation (Narendra and Parthasarathy, 1991), and the backpropagation trough time (Werbos, 1990). Unfortunately few stability studies were addressed considering the weights updating. Henriques et al (2001), based on Lyapunov stability theory, proposed a stable on-line learning law for the RNN based on the dual Kalman strategy, where both the hyper-state and the parameters are updated. To this aim it is assumed that the matrices $A$ and $C$ are static (off-line evaluated) and only the matrices $B$ and $D$ are to be updated on-line. The updating law is given by,

$$
\Delta W(k)=M(k)^{-1} \varphi(k)^{T} P A C \varepsilon(k)
$$

where $\varepsilon(k) \in \mathfrak{R}^{n_{y}}$ is the identification error $W(k) \in \Re^{n_{w}}=[\vec{B}(k) \vec{D}(k)]$ is a vector consisting of weights $\left(n_{w}\right)$ of matrices $B(k)$ and $D(k)$ at each time instant $k, A$ is assumed to be a Hurwitz matrix, 
$M(k) \in \mathfrak{R}^{n_{w} \times n_{w}}$ is given by (4) and $P \in \Re^{n \times n}$ is obtained from the discrete time $L$ yapunov equation (5).

$$
\begin{gathered}
M(k)=\left[I+\frac{1}{2} \varphi(k)^{T} P \varphi(k)\right] \\
A^{T} P A-P=-Q
\end{gathered}
$$

$\varphi(k) \in \mathfrak{R}^{n \times n_{w}}$ is an information matrix, based on $\sigma\left(x_{s}(k)\right)$ and $u(k) . Q \in \mathfrak{R}^{n \times n}$ is a user choice positive definite matrix and $I$ is an identity matrix of appropriate dimensions. Additionally, it is assumed that the hyper-state $x_{n}(k)$ is unknown and is to be determined by an observing procedure (Henriques et al, 2001). For this reason the pair $(A, C)$ is assumed observable. It is important to stress that, the network does not actually behave as an observer, in the strictest sense. In fact, it is not expected to estimate correctly the system state $x_{p}$ (which is assumed unknown), but only one possible representation in state space form $x_{n}$ for the system, such that the output of the neural model $y_{n}$ converge to the actual output plant $y$.

\section{NON-LINEAR OUTPUT REGULATION}

The OR problem for linear systems was solved by Francis (1977). For non-linear discrete time systems, Castillo et al (1993), using the zero output constrained algorithm (Monaco and Normand-Cyrot, 1987), has shown that the solution for the problem is reduced to the solution of transcendental non-linear equations, which represent the discrete time counterpart of the differential and transcendental equations, found for the continuous time systems by Isidori and Byrnes (1990).

\subsection{Problem Formulation}

Given a system in the form (1) and considering an additional external variable $w(k)$, the discrete time system is given by (6).

$$
\begin{gathered}
x(k+1)=f(x(k), u(k, w(k)) \\
y(k)=C x(k) \\
w(k+1)=s(w(k)) \\
e(k+1)=h(w(k), x(k))
\end{gathered}
$$

The vector $w \in \mathfrak{R}^{n_{y}}$ defines the disturbances and/or the reference signal generated by a so-called exosystem, and $e \in \Re^{n_{y}}$ defines the output tracking error. Given this extended system, it is desired to find conditions such that a controller in the form (7),

$$
u(k)=\gamma(x(k), w(k))
$$

where $\gamma: \Re^{n} \times \Re^{n_{y}} \rightarrow \Re^{n_{u}}$ is a smooth mapping satisfying the following two requirements.

S1: The equilibrium point $x=0$ of dynamics

$$
x(k+1)=f(x(k), \gamma(x(k), 0))
$$

S2: There exists a neighbourhood of the origin $(0,0)$ such that, for each initial state $(x(0), w(0))$, the solution of the closed loop system (9),

$$
\begin{gathered}
x(k+1)=f(x(k), \gamma(x(k), w(k)), w(k)) \\
w(k+1)=s(w(k))
\end{gathered}
$$

satisfies the error condition (10).

$$
\lim _{k \rightarrow \infty}(C x(k)-r(w(k)))=0
$$

where the desired output (reference) is generated by the exosystem

$$
y_{d}(k)=r(w(k))
$$

Castillo et al (1993) have shown that the state feedback discrete time regulator problem is locally solvable if there exist two mappings $x=\pi(w)$ and $u=c(w)$, satisfying (12).

$$
\begin{gathered}
\pi(s(w))=f(\pi(w), c(w), w) \\
0=C \pi(w)-r(w)
\end{gathered}
$$

Once evaluated the mappings $x=\pi(w)$ and $u=c(w)$, it is easy to show that the particular control law given by (13), satisfies both requirements S1 and S2.

$$
u(k)=\gamma(x, w)=c(w)+K(x-\pi(w))
$$

$K$ is a matrix of appropriate dimensions that places the eigenvalues of the first order approximation of the non-linear state space model in desired locations. As given by equation (12), the solution of the output regulator problem is reduced to a set of non-linear difference equations, known as regulator equations.

\subsection{Solution of Regulator Equations}

Except in a very few cases, it is difficult to derive an analytical solution to the mappings $x=\pi(w)$ and $u=c(w)$, that solves the regulator equations. One possibility is to solve approximately the regulator equations. Castillo et al (1993) have presented and derived conditions for the existence of an approximate solution for the discrete time case based on a polynomial expansion. Based on a Taylor series expansion as well, (Huang and Rugh, 1992) have proposed an approximation method for the continuous case. The same authors have presented an alternative approximation (Huang and Rugh, 1999) using a type of RNN, analogous to a cellular network. With a correct choice of parameters, the RNN is able to solve the regulator equations, in the least square sense, by means of a gradient descent minimisation.

B ased on a class of RNN, Henriques et al (2000), have proposed an approximation method to solve the regulator equations. The proposed algorithm leads to a pole placement design ensuring that the solution to the regulator equations converges if the eigenvalues of a given matrix are chosen to be stable.

\subsection{A daptive Control Structure}

The block diagram of the proposed control structure is shown in Fig. 3. Based on the identification error,

is locally exponentially stable; 
$e_{n}(k)=y_{n}(k)-y(k)$ the learning law (section 2) updates the neural parameters, $W$, and states, $x_{n}$.

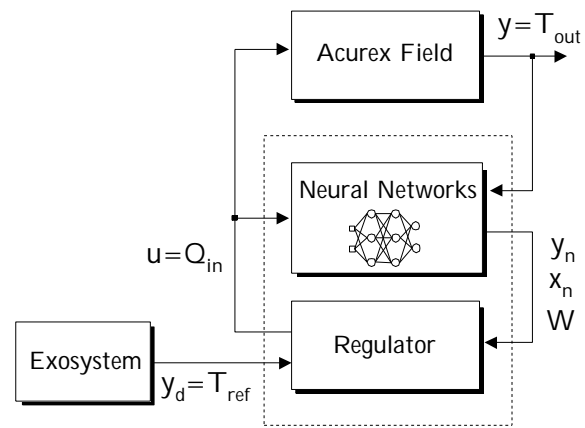

Fig. 3: Proposed control structure.

The output regulator design for the $\mathrm{NN}$ ensures the asymptotic convergence of the neural tracking error, $e_{d}(k)=y_{n}(k)-y_{d}(k)$. If the parameters of the neural model are adapted in the presence of parametric variations or uncertainties in the plant dynamics, the system tracking error will converge to zero. In fact, the system tracking error, $e(k)=y(k)-y_{d}(k)$, can be written as (14)

$$
e(k)=y(k)-y_{n}(k)+y_{n}(k)-y_{d}(k)
$$

Since the regulator assures (15)

$$
\lim _{k \rightarrow \infty}\left(y_{n}(k)-y_{d}(k)\right)=0
$$

the overall error will converge provided that the identification error also converges, i.e, (16).

$$
\lim _{k \rightarrow \infty}\left(y(k)-y_{n}(k)\right)=0
$$

\section{EXPERIMENTAL RESULTS}

The experiments were carried out in the Acurex Solar Collectors Field of the Plataforma Solar de Almería on 14 and 15 June 2001. The proposed control was implemented in C code and operates over a software developed at PSA (López, 1996) also in C code. The effectiveness of the developed approach was first tested using a non-linear distributed parameter model of the Acurex field, developed at the University of Sevilla (Berenguel et al, 1993). The sampling time was 15 seconds and the output temperature $\left(T_{\text {out }}\right)$ was considered as the maximum temperature of all the loops (another usual strategy is to assume the average value).

\subsection{Off-line Learning}

The distributed solar collector field is a process where the main disturbances, the solar radiation and the inlet oil temperature, are measurable. Therefore, it makes sense to use this knowledge in the design of a feedforward compensator. In the present work the relation (17), characterising the steady state behaviour, was used.

$$
Q_{i n}=\frac{11423 \times 10^{2} I_{r r}}{\left(903-0.67 T_{r e f}\right)\left(1820+3.47 T_{r e f}\right)\left(T_{\text {out }}-T_{\text {in }}\right)}
$$

A schematic diagram of the compensator is shown in Fig. 4.



Fig. 4: F eedforward compensator.

To obtain an initial estimation for the neural network parameters a number of test inputs were considered. The goal in designing the test inputs was to cover the operational range of the plant to as great and extend as possible. The number of training patterns, hidden neurons and input sequence are all chosen by an trial and error approach since there is still no reliable method available for determining these parameters systematically. It was found that a selection of two hidden neurons, $n=2$, is suitable to obtain a good model for the Acurex plant. As mentioned, the Levenberg-M arquardt algorithm was applied to obtaining an initial value for the matrices $A, B, C$, and $D$, defined in (2). Fig. 5 shows the off-line modelling results considering the experimental data set collected on 14 J une 2001.

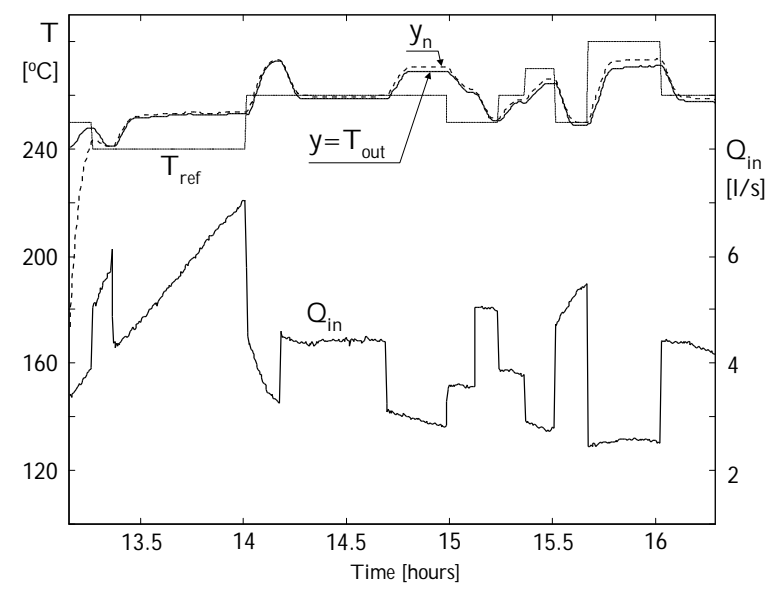

(a) Actual output versus neural output.

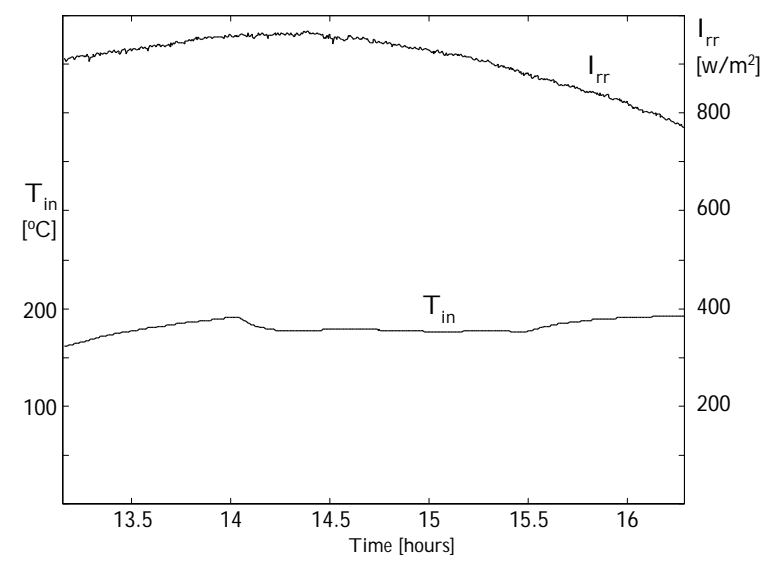

(b) Solar radiation and inlet oil temperature.

Fig. 5: Off-line neural modelling (14 J une 2001). 
In Fig. 5 the good performance of the learning methodology is clearly illustrated. As can be seen the output of the neural model $y_{n}$ performs considerably well in tracking the actual output $y=T_{\text {out }}$.

\subsection{Experimental results}

The present experiment was carried out on $15 \mathrm{~J}$ une 2001 and is intended to show the behaviour of the control system when several changes in the operation point, by setting different reference temperatures, are introduced. The effect of strong disturbances caused by large passing clouds, which produce drastic changes in the direct solar radiation level was tested, as well as the effect of inlet oil temperature variation.

As can bee seen in Fig. 6 the control behaviour is quite acceptable. The response presents almost no oscillations neither overshoots and after an initial transient phase the outlet oil temperature stabilises close to the reference.

The disturbance rejection capabilities of the controller are also acceptable, shown by a change in the inlet oil temperature, carried out at instant $15 \mathrm{~h} 00 \mathrm{~m}$.

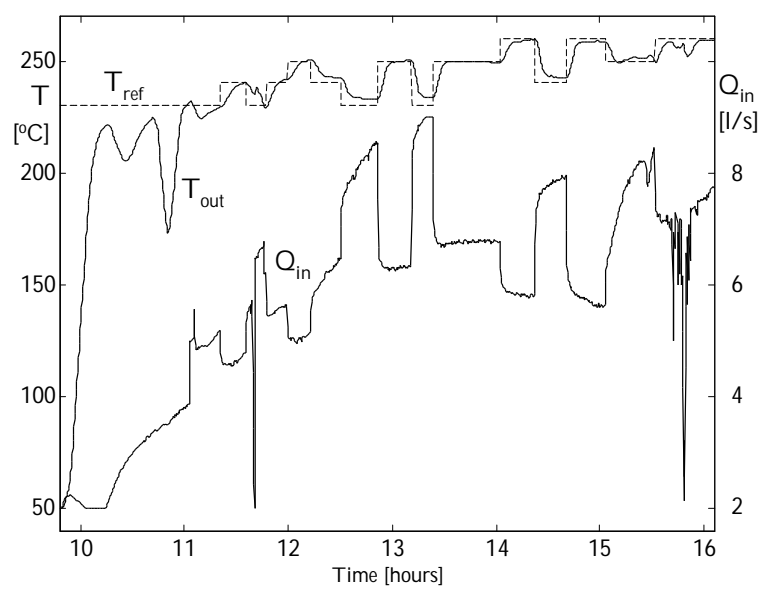

(a) Reference, output temperature and pump flow rate.

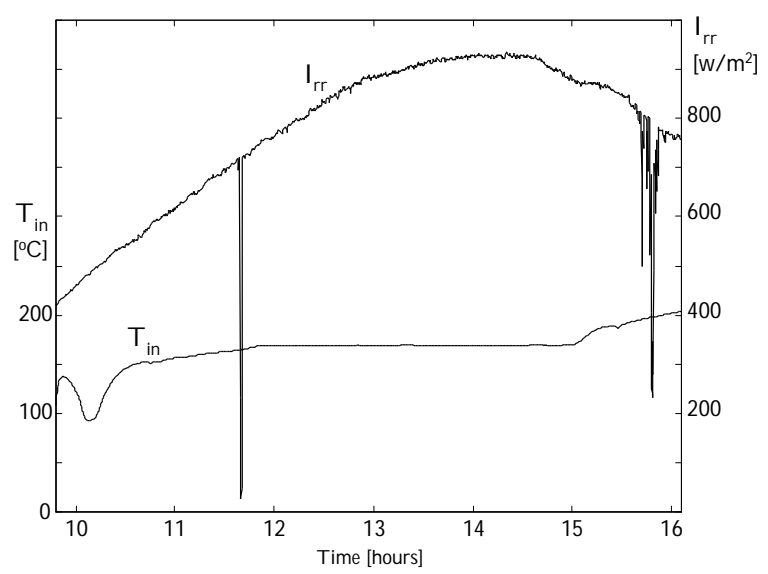

(b) Solar radiation and inlet oil temperature.

Fig. 6: Experimental results obtained on 15 J une 2001.

Although, it should be expected a zero steady state error. The actual state steady control error is justified by the lower gain characteristics of the on-line learning procedure. Since it was not possible to adjust during operation both learning and controller parameters their choice were not the most favourable. In future experiments it is expected to improve the learning procedure by increasing the learning gain.

In Fig. 6 the behaviour of the controlled system when intermittent clouds occurred (11h50m and $15 \mathrm{~h} 40 \mathrm{~m}$ ) can also be analysed. They produce changes in solar radiation that disturb the outlet oil temperature level during the operation. As observed the control results are very acceptable in this situation.

F rom several simulations, tested using the non-linear distributed parameter model of the Acurex field, (Berenguel et al, 1993), together with the experiments it can be concluded that the output regulation neural strategy performs according to its design: by on-line adjusting the neural parameters it is possible to reduce gradually model plant mismatches contributing to the convergence of tracking error steady-state offsets to zero. Moreover, it provides a control law such that the closed loop system is stable.

\section{CONCLUSIONS}

A non-linear control scheme based on a recurrent neural network has been implemented in real-time and applied to a distributed collector field in a solar power plant. The process is characterised by different operating conditions, depending on the changes in dynamics caused by variations in the solar radiation, reference temperature and plant characteristics.

The proposed strategy is a systematic one, which can be easily applied to a wide variety of processes with a small initial knowledge of the plant model. To cope with the inaccuracy of the off-line estimated neural parameters and possible changing dynamics, an adaptive strategy was employed providing an on-line scheme, ensuring stability and convergence properties. In this sense, the neural model can adaptively learn the system uncertainties and the regulator law adjusts the control action in order to guarantee a robust asymptotic error convergence.

Experimental results confirm the simulation results and show that the system has robustness with respect to changes in solar radiation, inlet oil temperature and operating conditions. This experimental study has shown that neural networks are an important methodology for many industrial control applications. The simplicity and reliability of neuro-control gives high potential for the development of efficient and intelligent control systems.

\section{ACK NOWLEDGEMENT S}

This work is partially supported by Project ALCINE Praxis/EEl/14155/98 and POSI Programa Operacional Sociedade de Informação of P ortuguese F undação para a Ciência e Tecnologia and European Union FEDER The experiments described in this paper were carried out within the project Improving Human Potencial Program (EC-DGXII) supported by the European Union Program Training and Mobility of Researchers. The authors would like to express their gratitude to the personnel of the PSA, in particular Diego M artinez and Loreto $V$ alenzuela, who were nominated to take care of this project. 


\section{REFERENCES}

Arahal, R., M. Berenguel, E. Camacho (1997), Nonlinear neural model based-predictive control of a solar plant, European Control Conference, ECC97, Bruxels, 1-4 J uly.

Berenguel, M., E. Camacho, F. Rubio (1993), Simulation Software Package for the Acurex, Internal Report, Sevilla.

Berenguel, M., E. Camacho, F. Rubio, P. Luk (1997), Incremental fuzzy $\mathrm{PI}$ control of a solar power plant, IEE Proc. Control Theory and Applications, 144, 6 596-604.

Camacho, E., M. Berenguel, F. Rubio (1994), Application of a gain scheduling generalised predictive controller to a solar power plant, Control Engineer Practice, 2, 2, 227-238.

Camacho, E., F. Rubio, F. Hughes (1992), Self-T uning control of a solar power plant with a distributed collector field, IEEE Control Systems Magazine, 12, 2, 72-78.

Cardoso, A., J. Henriques, A. Dourado (1999), F uzzy supervisor and feedforward control of a solar power plant using accessible disturbances, ECC99, 5rd European Control Conference, K arlsruhe, Germany.

Castillo, B., S. DiGennaro, D. Normand-Cyrot (1993), Non-linear regulation for a class of discrete time systems, Systems C ontrol L etters, 20, 57-65.

Coito, F., J. Lemos, R. Silva, E. Mosca (1997), A daptive control of a solar energy plant: Exploiting accessible disturbances, Int. Journal of Adaptive Control and Signal Processing, 11, 4, 327-342.

Cybenko, G. (1989), Approximation by Superposition of a Sigmoidal $\mathrm{F}$ unction, $\mathrm{M}$ athematics of $\mathrm{C}$ ontrol, Signals and Systems, 2, 3, 303-314.

Francis, B. (1997), The linear multivariable regulator problem, SIAM J. Control Optim., 15, 484-505.

Hagan, M. and B. Menhaj (1994), Training feedforward networks with the Marquardt algorithm, IEEE Trans. on Neural Networks, 5, 6, 989-993.

Henriques, J., A. Cardoso, A. Dourado, (1999), Supervision and C-M eans Clustering of PID Controllers for a Solar Power Plant, International J ournal of A pproximate Reasoning, 22, 73-91.

Henriques, J., B. Castillo, A. Titli, P. Gil, A. Dourado (2000), A Recurrent Neural Approach for The NonLinear Discrete Time output regulation, IEEE International Conference on Industrial Electronics, Control and Instrumentation-IECON, Nagoya, J apan.

Henriques, J., P. Gil, A. Dourado (2001), State space neural networks in non-linear adaptive system identification and control, IFAC Workshop on Advanced Neural-Fuzzy Control, Valencia, Spain, October 15-16.

Hopfield, J. (1982), Neural Networks and Physical Systems with Emergent Collective Computational A bilities, National A cademy of Sciences, 79, 25542558, USA.

Huang, J., and W. Rugh (1999), Stabilization on zeroerror manifolds and the non-linear servomechanism problem, IEEE Trans. A utom. Control, 37, 7, 10091003.
Huang, J. and W. Rugh (1992), An approximation method for the non-linear servomechanism problem, IEEE Trans. A utom. Control, 37, 9, 1395-1398.

Hunt, K. and R. Zbikowski (1997), Applications of Neural Adaptive Control Technology, World Scientific Series in Robotics and Intelligent Systems, Vol. 17.

Isidori, A. and C. Byrnes (1990), Output regulation for non-linear systems, IEEE Trans. A utomat. Control, 26, 131-140.

J in, L., M. Gupta, P. Nikiforuk (1999), Dynamic recurrent neural networks for approximation of nonlinear systems, 14th W orld Congress IF AC, 45-50, B eijin, China.

Kaltz, A. (1982), Distributed Collector System Plant Construction Report, IEA/SSPS Operating Agent DFVLR, Cologne, Neural Networks for Chemical Engineers, Editor A. Bulsari, Chap. 23, 572-591.

Kulawski, G. and M. Brdys (2000), Stable Adaptive Control with Recurrent Networks, A utomatica, 36, 522.

López, J. (1996), Acurex Filed D.A.S. and Control System, Internal Report 08/96, Plataforma Solar de A lmería.

Monaco, A. and D. Normand-Cyrot (1987), M inimum phase non-linear discrete time systems and feedback stabilization, Proc. 23th Conference on Decision and Control, Los Angeles, 979-986.

Narendra K. and K. Parthasarathy (1991), Gradient methods for the optimisation of dynamical systems containing neural networks, IEEE Trans. on Neural Networks, 2, 2, 252-262.

Oksanen, P. and E. J uuso (1999), A dvanced Control for Solar Systems at PSA, Proceedings of TOOLMET'99 Symposium Tool Environments and Development Methods for Intelligent System, O ulu, F inland, 123-134.

Pickhardt, R. and R. Silva (1998), A pplication of a nonlinear predictive controller to a solar power plant, Proceedings of the IEEE Conference on Control A pplications, Trieste.

Poznyak, A., W. Y u, E. Sanchez, P. Perez (1999), Nonlinear Adaptive Tracking Using Dynamic Neural Networks, IEEE Transactions on Neural Networks, 10, 6, 1402-1411.

Rovithakis, G. and M. Christodoulou (1997), Neural Adaptive Regulation of Unknown Non-Linear Dynamical Systems, IEEE Transactions on Systems, $M$ an and $C$ ybernetics, Part B, 27, 5, 810-822.

Rubio, F., M. Berenguel, E. Camacho (1995), Fuzzy logic control of a solar power plant, IEEE Transactions on F uzzy Systems, 3, 4, 459-468.

W erbos, P. (1990), Backpropagation through time: what it does and how to do it, Proceedings of the IEEE, 78, 10, 1550-1560.

W illiams, R. and D. Zipser (1995), Gradient-based learning algorithms for recurrent networks and their computational complexity. Backpropagation: Theory, architectures and applications, $Y$. Chauvin and D. Rumelhart Eds, Chap.13, 433-486. 